\title{
Aspirin is associated with an increased risk of subdural hematoma in normal-pressure hydrocephalus patients following shunt implantation
}

\author{
Peter Birkeland, MD, ${ }^{1}$ Jens Lauritsen, MD, PhD, ${ }^{2,3}$ and Frantz Rom Poulsen, MD, PhD ${ }^{1,3}$ \\ Departments of ${ }^{1}$ Neurosurgery and ${ }^{2}$ Orthopedic Surgery, Odense University Hospital; and ${ }^{3}$ Institute of Clinical Research, \\ University of Southern Denmark, Odense, Denmark
}

\begin{abstract}
OBJECT In this paper the authors investigate whether shunt-treated patients with normal-pressure hydrocephalus receiving aspirin therapy are at increased risk of developing subdural hematoma (SDH).

METHODS Records from 80 consecutive patients who had undergone implantation of a cerebrospinal fluid shunt for the treatment of normal-pressure hydrocephalus were retrospectively reviewed.

RESULTS Eleven cases of symptomatic SDH occurred, all among patients receiving aspirin or clopidogrel. The 5-year survival estimate was 0.3 ( $p<0.0001)$ for users of aspirin and the hazard ratio was $12.8(95 \% \mathrm{Cl} 3.1-53)$.

CONCLUSIONS Patients on an aspirin therapy regimen have a markedly increased risk of SDH after a shunt has been implanted for the treatment of normal-pressure hydrocephalus. Users of clopidogrel may have an even greater risk.

http://thejns.org/doi/abs/10.3171/2014.11.JNS14804
\end{abstract}

KEY WORDS subdural hematoma; normal-pressure hydrocephalus; aspirin; cerebrospinal fluid shunt; traumatic brain injury

$\mathrm{I}$ $\mathrm{N}$ neurosurgical practice, it is well recognized that patients admitted for subdural hematoma (SDH) often use antiplatelet medication, implying a causal relationship. ${ }^{1,6,13,14}$ However, a recent meta-analysis failed to show a significant association between the use of aspirin (acetylsalicylic acid) and the occurrence of SDH. ${ }^{5}$ Another frequent cause of referral to neurosurgical departments is normal-pressure hydrocephalus (NPH), which is recognized as a partly treatable cause of dementia. ${ }^{3}$ The condition is usually diagnosed in patients of increased age ${ }^{3}$ and these patients often receive antiplatelet medication as secondary prophylaxis therapy. There are case reports in the literature regarding $\mathrm{SDH}$ after shunt placement for NPH. ${ }^{4,8-10}$ In clinical series, this complication occurs in $5 \%-17 \%$ of cases. ${ }^{2,12,15}$ Previously, this complication has been studied in relation to lumbar puncture opening pressure, ${ }^{12}$ type of shunt valve, ${ }^{15}$ and long-term anticoagulation therapy. ${ }^{7}$ Systematic studies are, however, lacking. With the present study we aimed to investigate whether shunt-treated patients with NPH receiving aspirin therapy are at increased risk of developing SDH. To our knowledge, this study is the first to describe such a correlation.

\section{Methods}

Charts for all patients discharged with a primary diagnosis of NPH (ICD-10 diagnostic code G91.2) from the Department of Neurosurgery, Odense University Hospital, during the period from September 1, 2001, until March 31, 2013, were reviewed. Odense University Hospital provides neurosurgical health care services for a population of 1.3 million inhabitants. Until 2007, NPH had been diagnosed based on the presence of 2 or more of the triad of clinical findings (dementia, gait disturbance, and urinary incontinence), a brain scan suggesting hydrocephalus, and intracranial pressure monitoring. Since 2008, a lumbar perfusion test has been added to the diagnostic workup (modified from Katzman and Hussey ${ }^{11}$ ).

ABBREVIATIONS NPH = normal-pressure hydrocephalus; $\mathrm{SDH}=$ subdural hematoma

SUBMITTED April 16, 2014. ACCEPTED November 6, 2014.

INCLUDE WHEN CITING Published online January 2, 2015; DOI: 10.3171/2014.11.JNS14804.

DISCLOSURE Dr. Poulsen is part of the Danish Study Group for Subdural Hematoma. The authors report no conflict of interest concerning the materials or methods used in this study or the findings specified in this paper. 
If the diagnosis of NPH was confirmed, the patient was referred for implantation of a ventriculoperitoneal shunt or, in case of peritoneal adhesions from previous surgery, a ventriculoatrial shunt.

Various shunt devices and valve settings for NPH have been used, such as the Delta valve and Strata programmable valve (both Medtronic), and Codman Hakim and Certas programmable valves (both DePuy). Customarily, the Codman valves were initially set with a high resistance, which was gradually decreased. Strata valves were generally set at a performance level of 1.0 from the time of implantation.

Antiplatelet and anticoagulation therapies are prescribed for secondary prevention in ischemic heart disease, after ischemic stroke/transient ischemic attack, and in aortic or peripheral vascular disease. Aspirin, clopidogrel, dipyridamole, and ticagrelor are given at a dosage of $75-150 \mathrm{mg}$ once daily, $75 \mathrm{mg}$ once daily, $200 \mathrm{mg}$ twice daily, and $90 \mathrm{mg}$ twice daily, respectively. The administration of warfarin follows an algorithm based on international normalized ratio monitoring. If patients were receiving antiplatelet and/or anticoagulation therapy, the therapy was routinely discontinued for 7 days prior to and resumed 1-2 days after surgery. Patients were routinely referred for a brain CT scan 1 month postoperatively to rule out asymptomatic hygroma. If a hygroma was found, a programmable valve was adjusted. The following information was extracted from the patient charts: birth date, sex, referral data, date of surgery, use and dosage of antiplatelet and anticoagulant medication, subsequent admission for symptomatic SDH (if applicable), date of shunt removal (if applicable), and date of death (if applicable) or date of case review, whichever came first. To avoid reporting bias, information regarding drug use was based on the full hospital record for all types of admissions in all specialties and not only on information given in case of readmission. If the records indicated that the patient did not take the antiplatelet or anticoagulation medication during the complete study period, the patient was recorded as a nonuser. The term "symptomatic SDH" included acute and chronic SDH and was defined by the clinical decision to intervene by external regulation of the shunt valve (if possible), valve replacement surgery, bur hole placement (in case of chronic SDH), or craniotomy (for acute SDH). The data were recorded using the EpiData Entry Software (www.epidata.dk). The process of extracting and recording data was done twice, and the data were stored in 2 separate files. Any case of mismatch was detected using the double entry verification feature of the software and settled by reviewing the patient chart. Finally, a single validated data file was created. The study was approved by The Danish Data Protection Agency.

\section{Statistical Analysis}

Data were analyzed using EpiData Analysis Software (www.epidata.dk). Hazard ratios, 5-year survival estimates, and 95\% confidence intervals were calculated. Because of the low number of patients and need for simplification, comparison of survival in separate analysis for the following groups was chosen: base level (no antiplatelet or anticoagulation medication, $n=41$ ) versus aspirin in com- bination with other antiplatelet or anticoagulation drugs (n $=35$ ) or aspirin excluding clopidogrel and warfarin ( $\mathrm{n}=$ $31)$ or clopidogrel, including concomitant aspirin $(n=5)$.

\section{Results}

Between September 1, 2001, and March 31, 2013, ventriculoperitoneal shunts were implanted in 78 patients and ventriculoatrial shunts were implanted in 2 patients. There were 56 men and 24 women. The mean age at the time of surgery was 70 years (range 47-84 years). One patient received a valveless shunt. Thirty-nine patients received a Medtronic Delta unishunt (performance levels 1.0 [31 patients], 1.5 [6 patients], or 2.0 [2 patients]). Forty patients were given shunts with adjustable valves: Medtronic Strata (11 patients), Codman Hakim (27 patients), and Codman Certas (2 patients). Aspirin was used by 35 patients; thus, it was the most commonly used antiplatelet or anticoagulant medication. Thirteen of these patients used a second drug: dipyridamole (8 patients), clopidogrel (2 patients), warfarin (2 patients), and ticagrelor (1 patient). Additionally, 3 patients received clopidogrel only and 1 patient warfarin only. The mean age among users of aspirin was 72.5 years (range 55-84 years) as opposed to 65.7 years (range 47-81 years) among patients not receiving antiplatelet or anticoagulant medication.

Patients were followed up for a mean of 938 days (median 897 days, range 117-2431 days). Eleven of the 80 patients (14\%) were treated for SDH; 9 of these 11 patients were male. These 11 patients had a valveless shunt (1 patient), a Medtronic Delta 1.0 unishunt (5 patients), a Medtronic Strata shunt (3 patients), and a Codman Hakim shunt (2 patients) at the time of admission for SDH. Subdural hematomas exclusively occurred in patients taking antiplatelet medication. Nine of the patients were receiving either aspirin monotherapy ( $75 \mathrm{mg}$ once daily) or aspirin $(75 \mathrm{mg}$ once daily) combined with dipyridamole (2 patients), warfarin (1 patient), or ticagrelor (1 patient). The remaining 2 patients were receiving clopidogrel monotherapy. The 5-year survival estimate for aspirin users was $0.3(p<0.0001$; Table 1). Correspondingly, the hazard ratio was 12.8 (95\% CI 3.1-53). Figure 1 depicts a Kaplan-Meier plot of eventfree survival (an event was defined as the occurrence of an SDH). It is noteworthy that SDHs occurred throughout the study period. For clopidogrel users, the 5-year survival estimate and hazard ratio was $0.4(\mathrm{p}<0.0001)$ and 433 (95\% CI $10.2-18,300)$, respectively. We were not able to give estimates regarding the use of warfarin because of the limited number of patients. Thirteen deaths occurred during the study period, 6 among patients not receiving antiplatelet or anticoagulation medication and 7 among those receiving aspirin. No deaths were attributed to the occurrence of an SDH.

\section{Discussion}

We analyzed clinical data from 80 consecutive patients who had a ventriculoperitoneal shunt $(n=78)$ or ventriculoatrial shunt $(n=2)$ for treatment of NPH with the purpose of estimating risk for SDH in relation to antiplatelet medication. Subdural hematoma occurred in $14 \%$ of cases (11 patients), which is comparable to previous reports. ${ }^{2,12,15}$ 
TABLE 1. Survival estimates and hazard ratios for SDH

\begin{tabular}{|c|c|c|c|c|c|c|c|c|c|}
\hline \multirow{2}{*}{$\begin{array}{c}\text { Antiplatelet or } \\
\text { Anticoagulation Medication }\end{array}$} & \multirow{2}{*}{$\begin{array}{l}\text { No. of } \\
\text { Pts }^{*}\end{array}$} & \multirow{2}{*}{$\begin{array}{l}\text { No. of Pts } \\
\text { w/ SDH }\end{array}$} & \multirow{2}{*}{$\begin{array}{l}\text { No. of Pts } \\
\text { Who Died }\end{array}$} & \multicolumn{3}{|c|}{ Observation Time (days) } & \multirow{2}{*}{$\begin{array}{c}\text { 5-Yr Survival } \\
\text { Estimate }(95 \% \mathrm{Cl})\end{array}$} & \multirow[b]{2}{*}{$p$ Value } & \multirow[b]{2}{*}{$\mathrm{HR}(95 \% \mathrm{Cl})$} \\
\hline & & & & Minimum & Maximum & Total & & & \\
\hline None & 41 & 0 & 6 & 203 & 2431 & 39,551 & $1.0(1.0-1.0)$ & & 1 (reference) \\
\hline ASA & 35 & 9 & 7 & 117 & 1996 & 29,840 & $0.3(0.0-0.6)$ & $<0.0001$ & $10.8(2.9-41.0)$ \\
\hline $\begin{array}{l}\text { ASA w/o clopidogrel or } \\
\text { warfarin }\end{array}$ & 31 & 8 & 7 & 117 & 1996 & 27,270 & $0.3(0.0-0.6)$ & $<0.0001$ & $12.8(3.1-53.0)$ \\
\hline Clopidogrel & 5 & 2 & 0 & 212 & 1965 & 4538 & $0.4(-0.2$ to 0.9$)$ & $<0.0001$ & $433(10.2-18,300)$ \\
\hline
\end{tabular}

ASA = acetylsalicylic acid (aspirin); Pts = patients.

* Patients taking both aspirin and clopidogrel are listed twice. The patient taking warfarin only is not listed.

We found a significant increased risk of SDH among users of aspirin compared with nonusers. This differs from the results of a recent meta-analysis of randomized clinical trials regarding antiplatelet therapy for the primary or secondary prevention of stroke, where no increased risk of SDH in patients using aspirin was identified. ${ }^{5}$ The differing patient populations may explain this discrepancy. We hypothesize that shunting for NPH may render the brain prone to collapse, which in combination with antiplatelet therapy can result in increased susceptibility to the development of an SDH.

The study, however, has limitations. First, it is a singlecenter experience with a relatively small patient sample size. Second, during the study period various shunts and settings have been in use. Third, the simplification of antiplatelet medication to focus on aspirin only may not fully explain risks in relation to other medications, particularly new and combined drugs. Among patients in the present study we found only 5 users of clopidogrel (2 in combination with aspirin) and 1 user of ticagrelor (in combination with aspirin). We chose to give a separate estimate for clopidogrel as indicated in Table 1, since there were no SDHs among the 2 users of aspirin and clopidogrel in combination. However, due to the very small number of patients, it is not possible to estimate a possible additive risk of effect of the risk of SDH associated with a combination of drugs.

Fourth, there may be several confounders at work. It is not possible to separate the effect of antiplatelet agents with the underlying comorbidity leading to antiplatelet therapy (bias by indication). This bias can only be eliminated in a randomized controlled trial, but there would have been another way to address it. If we had run antiplatelet assays, we could potentially have identified nonresponders to aspirin or clopidogrel therapy in our patient sample. This additional information could have helped us to strengthen a causal relationship between the medication and the formation of a symptomatic SDH. However, antiplatelet assays were only recently introduced in our practice, which precludes us from presenting such data. Age is another confounder. Users of aspirin were on average 7 years older than nonusers. This may explain a slightly increased risk but, in our opinion, not the markedly increased risk seen. There was no difference in the type of shunt used in patients who suffered SDH and those who did not. Information regarding drug use was based on hospital records. We deliberately chose not to rely solely on information given at the time of admission for SDH to

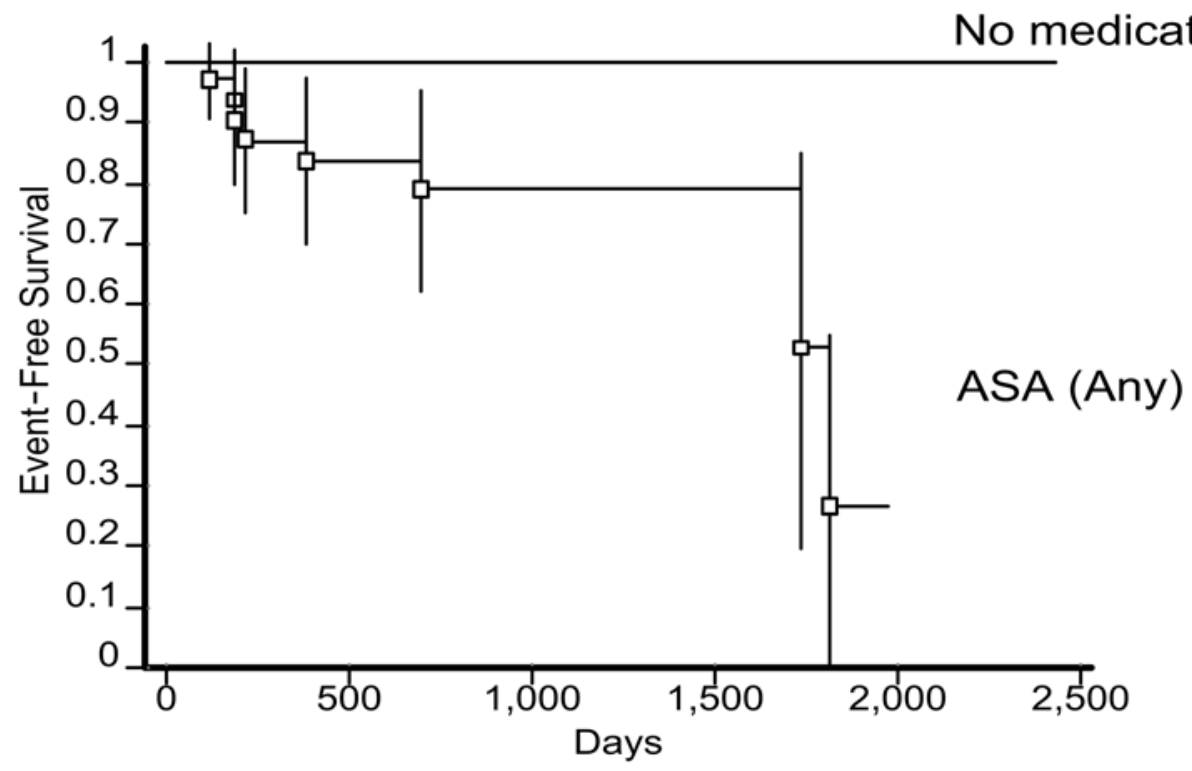

FIG. 1. Kaplan-Meier plot of event-free survival (an event was defined as the occurrence of an SDH). ASA = acetylsalicylic acid (aspirin). 
avoid reporting bias. There may be a medication compliance bias, but this is likely to be minor, as the cases all reported the use of antiplatelet therapy at the time of admission for SDH. Some patients might have been treated for SDH at another hospital. However, the referral policy in our region implies that all neurosurgical problems lead to admission to our department, but a few patients may have migrated to a different region. Finally, we did not collect data on functional outcome in the present study. Some of the limitations could be addressed by a countrywide registry-based study.

This study does not determine whether the underlying comorbidity leading to aspirin therapy contributes to the increased risk seen; however, we find it unlikely to play a major role. Presently, we do not recommend that patients undergoing shunt treatment for NPH should stop receiving aspirin. We routinely withhold aspirin and other antiplatelet agents 7 days prior to surgery and resume it 1-2 days postoperatively to reduce the risk of perioperative and immediate postoperative bleeding. We would suggest that the increased risk should be discussed with the patient prior to shunt implantation.

\section{Conclusions}

Despite these limitations, our data from a consecutive series of 80 patients from a confined region of 1.3 million inhabitants show that users of aspirin who have a shunt to treat NPH have a markedly increased risk of SDH compared with nonusers. Users of clopidogrel may have an even greater risk.

\section{References}

1. Aspegren OP, Åstrand R, Lundgren MI, Romner B: Anticoagulation therapy a risk factor for the development of chronic subdural hematoma. Clin Neurol Neurosurg 115:981-984, 2013

2. Baldini M, Princi L, Vivenza C: Subdural hematoma secondary to CSF ventricular shunt. Schweiz Arch Neurol Neurochir Psychiatr 127:5-14, 1980

3. Brean A, Eide PK: Prevalence of probable idiopathic normal pressure hydrocephalus in a Norwegian population. Acta Neurol Scand 118:48-53, 2008

4. Chrissicopoulos C, Mourgela S, Kirgiannis K, Sakellaropoulos A, Ampertos N, Petritsis K, et al: What is the appropriate shunt system for normal pressure hydrocephalus? Acta Neurochir Suppl 113:119-121, 2012

5. Connolly BJ, Pearce LA, Kurth T, Kase CS, Hart RG: Aspirin therapy and risk of subdural hematoma: meta-analysis of randomized clinical trials. J Stroke Cerebrovasc Dis 22:444-448, 2013
6. De Bonis P, Trevisi G, de Waure C, Sferrazza A, Volpe M, Pompucci A, et al: Antiplatelet/anticoagulant agents and chronic subdural hematoma in the elderly. PLoS ONE 8:e68732, 2013

7. Goodwin CR, Kharkar S, Wang P, Pujari S, Rigamonti D, Williams MA: Evaluation and treatment of patients with suspected normal pressure hydrocephalus on long-term warfarin anticoagulation therapy. Neurosurgery 60:497-502, 2007

8. Gürer B, Yilmaz ER, Kertmen HH, Sekerci Z: Shunt overdrainage after mild head trauma. Clin Neurol Neurosurg 114:196-198, 2012

9. Hayes J, Roguski M, Riesenburger RI: Rapid resolution of an acute subdural hematoma by increasing the shunt valve pressure in a 63-year-old man with normal-pressure hydrocephalus with a ventriculoperitoneal shunt: a case report and literature review. J Med Case Reports 6:393, 2012

10. Kamano S, Nakano Y, Imanishi T, Hattori M: Management with a programmable pressure valve of subdural hematomas caused by a ventriculoperitoneal shunt: case report. Surg Neurol 35:381-383, 1991

11. Katzman R, Hussey F: A simple constant-infusion manometric test for measurement of CSF absorption. I. Rationale and method. Neurology 20:534-544, 1970

12. Khan QU, Wharen RE, Grewal SS, Thomas CS, Deen HG Jr, Reimer R, et al: Overdrainage shunt complications in idiopathic normal-pressure hydrocephalus and lumbar puncture opening pressure. J Neurosurg 119:1498-1502, 2013

13. Lindvall P, Koskinen LO: Anticoagulants and antiplatelet agents and the risk of development and recurrence of chronic subdural haematomas. J Clin Neurosci 16:1287-1290, 2009

14. O'Brien DF, Basu S, O'Donnell JR, Roberts GA, Phillips $\mathrm{J}$ : The impact of aspirin therapy and anticoagulation on the prevalence of spontaneous subdural haematoma. Ir Med J 93:244-246, 2000

15. Zeilinger FS, Reyer T, Meier U, Kintzel D: Clinical experiences with the dual-switch valve in patients with normal pressure hydrocephalus. Acta Neurochir Suppl 76:559-562, 2000

\section{Author Contributions}

Conception and design: Birkeland. Acquisition of data: Birkeland. Analysis and interpretation of data: all authors. Drafting the article: Birkeland. Critically revising the article: Lauritsen, Poulsen. Reviewed submitted version of manuscript: all authors. Approved the final version of the manuscript on behalf of all authors: Birkeland. Statistical analysis: Birkeland, Lauritsen. Study supervision: Poulsen.

\section{Correspondence}

Peter Birkeland, Department of Neurosurgery, Odense University Hospital, Sdr. Blvd. 29, DK-5000 Odense C, Denmark. email: peter@birkeland.dk. 